\title{
The Non-isentropic Perturbation of an Arbitrary Simple Wave
}

\section{ROY GUNDERSEN}

\author{
Communicated by L. Bers
}

1. Introduction. In a recent paper [1], a method was presented for discussing the perturbation of a given one-dimensional, non-steady flow, assumed isentropic, of an inviscid, ideal compressible gas. It was not assumed that the perturbed flow was isentropic. Explicit solutions were obtained for a basic flow which was uniform or a centered simple wave, but for an arbitrary simple wave only if the perturbed flow was isentropic. In the present paper, it is shown that the non-isentropic perturbation of an arbitrary simple wave can be solved by quadratures.

The equation of state is

$$
P=\exp \left\{\frac{s-s^{*}}{c_{v}}\right\} \rho^{\gamma}
$$

and the equations which characterize the flow are

$$
\begin{gathered}
c_{t}+u c_{x}+\frac{1}{2}(\gamma-1) c u_{x}=0, \\
u_{t}+u u_{x}+\frac{2 c c_{x}}{\gamma-1}-\frac{c^{2} s_{x}}{c_{v} \gamma(\gamma-1)}=0 \\
s_{t}+u s_{x}=0
\end{gathered}
$$

where $P$ is the pressure, $\rho$ the density, $s$ the specific entropy, $s^{*}$ the specific entropy at some reference state, $\gamma$ the ratio of the specific heats at constant pressure $c_{p}$ and at constant volume $c_{v}, u$ the particle velocity, $c$ the local speed of sound and the subscripts denote partial derivatives.

A formal linearization in the neighborhood of a known isentropic solution, denoted by the subscript zero, gives the following system of linear equations which govern the first approximation to the disturbed flow:

$$
\begin{gathered}
c_{1 t}+u_{0} c_{1 x}+\frac{1}{2}(\gamma-1) c_{0} u_{1 x}+u_{1} c_{0 x}+\frac{1}{2}(\gamma-1) u_{0 x} c_{1}=0, \\
\frac{1}{2}(\gamma-1) u_{1 t}+\frac{1}{2}(\gamma-1) u_{0} u_{1 x}+c_{0} c_{1 x}+\frac{1}{2}(\gamma-1) u_{0 x} u_{1}+c_{1} c_{0 x}=\frac{c_{0}^{2} s_{1 x}}{2 c_{v} \gamma},
\end{gathered}
$$




$$
s_{1 t}+u_{0} s_{1 x}=0,
$$

where the subscript one denotes the terms of first order.

According to (3), $s_{1}$ remains constant along the particle paths of the given flow, i.e., along $d x / d t=u_{0}$. Also, $\rho_{0}\left(d x-u_{0} d t\right)$ is the exact differential of a function $\psi_{0}$ which, when equated to a constant, defines the particle paths. Hence the solution of (3) is

$$
s_{1}=\omega\left(\psi_{0}\right)
$$

with $\omega$ an arbitrary function. It is convenient to define a new function $H_{0}(x, t)$ by

$$
c_{0}^{2} s_{1 x}=\rho_{0} c_{0}^{2} \omega^{\prime}\left(\psi_{0}\right)=\gamma(\gamma-1) c_{v} H_{0} .
$$

Thus the two equations which serve to determine $u_{1}, c_{1}$ are (1) and

$$
u_{1 t}+u_{0} u_{1 x}+\frac{2 c_{0} c_{1 x}}{\gamma-1}+u_{0 x} u_{1}+\frac{2 c_{0 x} c_{1}}{\gamma-1}=H_{0} .
$$

It is convenient to introduce the characteristic parameters of the basic flow

$$
\frac{1}{2} u_{0}+\frac{c_{0}}{\gamma-1}=\alpha, \quad-\frac{1}{2} u_{0}+\frac{c_{0}}{\gamma-1}=\beta
$$

and the functions

$$
\frac{1}{2} u_{1}+\frac{c_{1}}{\gamma-1}=A, \quad-\frac{1}{2} u_{1}+\frac{c_{1}}{\gamma-1}=B .
$$

The combinations $(1) /(\gamma-1) \pm \frac{1}{2}(5)$ give

$$
\begin{gathered}
A_{t}+\left(u_{0}+c_{0}\right) A_{x}+\frac{1}{2}[A(\gamma+1)+(\gamma-3) B] \alpha_{x}=\frac{1}{2} H_{0}, \\
B_{t}+\left(u_{0}-c_{0}\right) B_{x}+\frac{1}{2}[A(3-\gamma)-(\gamma+1) B] \beta_{x}=-\frac{1}{2} H_{0} .
\end{gathered}
$$

2. Formulation of the Problem. For the simple wave, one of the characteristic parameters $(\alpha, \beta)$ of the basic flow remains constant, e.g., $\beta=\beta_{0}$. $B$ will be determined from (7) and then $A$ from (6).

Let $\left(x_{0}(z), t_{0}(z)\right)$ be the parametric representation of a curvilinear characteristic of the arbitrary simple wave. The wave may then be represented by

$$
\begin{gathered}
x=x_{0}(z)+\left[u_{0}(z)+c_{0}(z)\right] \tau, \quad t=t_{0}(z)+\tau, \\
-\frac{1}{2} u_{0}(z)+\frac{c_{0}(z)}{\gamma-1}=\beta_{0} .
\end{gathered}
$$

The particle paths are given by $\rho_{0} c_{0} \tau=$ const. or $y \equiv c_{0}^{2} \tau^{2 \theta}=$ const., where $\theta=(\gamma-1) /(\gamma+1)$. From (4),

$$
\gamma(\gamma-1) c_{v} H_{0}=\left(\rho_{0} c_{0} \tau\right) \frac{c_{0}}{\tau} \omega^{\prime}(y),
$$


so that it is convenient to write

$$
H_{0}=2 \frac{c_{0}}{\tau} \Omega^{\prime}(y)
$$

where $\Omega$ is an arbitrary differentiable function.

3. Solution. Equation (7) becomes

$$
B_{t}+\left(u_{0}-c_{0}\right) B_{x}=-\frac{c_{0} \Omega^{\prime}(y)}{\tau}
$$

It is convenient to change from the independent variables $(x, t)$ to $(z, \tau)$ in (10). With $B(x, t) \equiv B_{1}(z, \tau)$, the result is

$$
\left(u_{0}^{\prime}+c_{0}^{\prime}\right) \tau B_{1 \tau}-2 c_{0} B_{1 z}=-\frac{J c_{0} \Omega^{\prime}(y)}{\tau},
$$

where the Jacobian has the value

$$
J=\frac{c_{0}^{\prime} \tau}{\theta}-2 c_{0} t_{0}^{\prime}
$$

and primes denote differentiation with respect to the argument. From the definition of the characteristic parameters, $u_{0}+c_{0}=c_{0} / \theta-2 \beta_{0}$, so that (11) becomes

$$
\frac{c_{0}^{\prime} \tau B_{1 \tau}}{\theta}-2 c_{0} B_{1 z}=-\frac{J c_{0} \Omega^{\prime}(y)}{\tau}
$$

The solution to (12) is obtained from the system

$$
\frac{\theta d \tau}{\tau \frac{d c_{0}}{d z}}=\frac{d z}{-2 c_{0}}=\frac{\tau d B_{1}}{-J c_{0} \Omega^{\prime}(y)}
$$

The following first integral is obtained from the first two ratios of (13):

$$
c_{0}=K \tau^{-2 \theta} \text { or } \rho_{0} c_{0} \tau^{2}=\text { const. }
$$

where $K$ is a constant. It will be seen that this is a convenient representation of the curvilinear characteristics of the wave.

From the last two ratios of (13)

$$
2 \frac{d B_{1}}{d z}=\left[\frac{\tau}{\theta} \frac{d c_{0}}{d z}-2 c_{0} \frac{d t_{0}}{d z}\right] \frac{1}{\tau} \Omega^{\prime}\left[c_{0}^{2} \tau^{2 \theta}\right]
$$

or

$$
2 d B_{1}=\frac{d c_{0}}{\theta} \Omega^{\prime}\left[c_{0}^{2} \tau^{2 \theta}\right]-\frac{2 c_{0}}{\tau} d t_{0} \Omega^{\prime}\left[c_{0}^{2} \tau^{2 \theta}\right]
$$

By the use of the first integral (14), (15) yields a second first integral: 


$$
B_{1}=\frac{\Omega\left[K^{2} \tau^{-2 \theta}\right]}{2 \theta K}+\int\left\{\frac{d}{d \tau} \Omega\left[K^{2} \tau^{-2 \theta}\right]\right\} \frac{d t_{0}}{2 \theta K} .
$$

The first integral (14) gives a relation between $z$ and $\tau$, so that an integration by parts transforms (16) to

$$
B_{1}=\frac{\Omega\left[K^{2} \tau^{-2 \theta}\right]}{2 \theta}-\frac{t_{0}^{\prime}}{c_{0}^{\prime}} \tau^{-2 \theta-1} \Omega\left[K^{2} \tau^{-2 \theta}\right]+\int \Omega\left[K^{2} \tau^{-2 \theta}\right] d\left[\frac{t_{0}^{\prime}}{c_{0}^{\prime}} \tau^{-2 \theta-1}\right] .
$$

From the two first integrals (14) and (17), the solution of equation (10) is

$$
\begin{aligned}
B_{1}=2\left(\rho_{0} c_{0}\right)^{\frac{3}{3}}\left(t-t_{0}\right) F^{\prime}\left[\rho_{0} c_{0}\left(t-t_{0}\right)^{2}\right]+ & \frac{c_{0} \Omega(y)}{2 \theta y} \\
& -\Omega(y)\left(t-t_{0}\right)^{-2 \theta-1} \frac{t_{0}^{\prime}}{c_{0}^{\prime}}+R(z, \tau),
\end{aligned}
$$

where $R(z, \tau)$ denotes the result obtained by performing the quadrature indicated in equation (17) and then replacing $K$ by $c_{0} \tau^{2 \theta}$ from the first integral (9), and $F$ is an arbitrary differentiable function.

The solution of (6) is obtained from the system

$$
d t=\frac{d x}{u_{0}+c_{0}}=\frac{2 d A}{H_{0}+[(3-\gamma) B-(\gamma+1) A] \alpha_{x}} .
$$

The integral of the first equation of (19) has the first family of characteristics (rectilinear characteristics) for level curves. Let this first integral be

$$
z(x, t)=a, \text { a constant. }
$$

This function is defined implicitly by

$$
x-x_{0}(z)=\left[u_{0}(z)+c_{0}(z)\right]\left[t-t_{0}(z)\right] .
$$

From the definition of the characteristic parameters

$$
(\gamma+1) \alpha=2\left[u_{0}+c_{0}-\frac{1}{2}(\gamma-3)\right] \beta_{0} .
$$

Substitution in (19) gives a differential equation for $A$. To integrate this equation, $B$ is substituted from (18) and the first integral (20) is utilized, i.e., $z$ may be treated as a constant. This equation is

$$
\begin{aligned}
\frac{d}{d t}\left[\left(t-t_{0}-\frac{2 \theta c_{0} t_{0}^{\prime}}{c_{0}^{\prime}}\right) A\right]-\frac{3-\gamma}{\gamma+1} \frac{d}{d t}\left\{\frac{F\left[\rho_{0} c_{0}\left(t-t_{0}\right)^{2}\right]}{\left(\rho_{0} c_{0}\right)^{\frac{1}{2}}}\right\} \\
-\frac{3-\gamma}{\gamma+1} R\left(a, t-t_{0}\right) \\
=\left(t-t_{0}-\frac{2 c_{0} \theta t_{0}^{\prime}}{c_{0}^{\prime}}\right)\left[\frac{c_{0} \Omega^{\prime}(y)}{t-t_{0}}+\frac{3-\gamma}{2(\gamma-1)} \frac{c_{0}}{y} \frac{\Omega(y)}{\left(t-t_{0}\right)}\right] .
\end{aligned}
$$

Integrating (21) and performing an integration by parts yields a second first integral: 


$$
\begin{aligned}
\left(t-t_{0}-\frac{2 c_{0} \theta t_{0}^{\prime}}{c_{0}^{\prime}}\right) A & =\frac{3-\gamma}{\gamma+1} \frac{F\left[\rho_{0} c_{0}\left(t-t_{0}\right)^{2}\right]}{\left(\rho_{0} c_{0}\right)^{\frac{1}{2}}} \\
+ & \frac{3-\gamma}{\gamma+1} \int R\left(a, t-t_{0}\right) d t+\frac{c_{0}}{2 \theta y} \Omega(y)\left(t-t_{0}-\frac{2 c_{0} \theta t_{0}^{\prime}}{c_{0}^{\prime}}\right) \\
& -\frac{1}{2 \theta c_{0}} \int \Omega(y) \frac{d}{d t}\left[\left(t-t_{0}-\frac{2 c_{0} \theta t_{0}^{\prime}}{c_{0}^{\prime}}\right)\left(t-t_{0}\right)^{-2 \theta}\right] d t \\
& +\frac{3-\gamma}{2(\gamma-1) c_{0}} \int \Omega(y)\left(t-t_{0}-\frac{2 c_{0} \theta t_{0}^{\prime}}{c_{0}^{\prime}}\right)\left(t-t_{0}\right)^{-2 \theta-1} d t .
\end{aligned}
$$

Hence the solution for $A$ is

$$
\begin{aligned}
\left(t-t_{0}\right. & \left.-\frac{2 c_{0} \theta t_{0}^{\prime}}{c_{0}^{\prime}}\right) A=\frac{3-\gamma}{\gamma+1} \frac{F\left[\rho_{0} c_{0}\left(t-t_{0}\right)^{2}\right]}{\left(\rho_{0} c_{0}\right)^{\frac{1}{2}}} \\
& +\frac{c_{0} \Omega(y)}{2 \theta y}\left(t-t_{0}-\frac{2 c_{0} \theta t_{0}^{\prime}}{c_{0}^{\prime}}\right)+G(z)+\left\{\frac{3-\gamma}{\gamma+1} \int R\left(a, t-t_{0}\right) d t\right. \\
& -\frac{1}{2 \theta c_{0}} \int \Omega(y) \frac{d}{d t}\left[\left(t-t_{0}-\frac{2 c_{0} \theta t_{0}^{\prime}}{c_{0}^{\prime}}\right)\left(t-t_{0}\right)^{-2 \theta}\right] d t \\
& \left.+\frac{3-\gamma}{2(\gamma-1) c_{0}} \int \Omega(y)\left(t-t_{0}-\frac{2 c_{0} \theta t_{0}^{\prime}}{c_{0}^{\prime}}\right)\left(t-t_{0}\right)^{-2 \theta-1} d t\right\}_{a=z},
\end{aligned}
$$

where the notation \{\}$_{a=z}$ indicates that the integrations are to be carried out with $z$ replaced by the constant $a$, and then $a$ is to be replaced by the variable $z$ to give the solution (23), and $G$ is an arbitrary function.

Equations (18) and (23) give the solution of equations (6) and (7) in terms of three arbitrary functions $F, G$ and $\Omega$. Theoretically these functions may be determined in an actual problem, as is easily seen by a generalization of the discussion given in paragraph 6 of [1].

To obtain the solution for a wave centered at $x=0, t=0$, let $z=x / t$ and $t_{0}=0$. This is seen to agree with results presented in [1].

This work was supported by a grant from the National Science Foundation.

\section{ReFERENCE}

[1] R. M. Gundersen, The flow of a compressible fluid with weak entropy changes, J. Fluid Mech. 3 (1958). 